\title{
Single-center study on transplantation of livers donated after cardiac death: A report of 6 cases
}

\author{
XU-YONG SUN ${ }^{1-3}, J_{A N-H U I ~ D O N G}{ }^{1}$, KE QIN $^{1}$, LIU-GEN LAN ${ }^{1}$, HAI-BIN LI ${ }^{1}$, \\ YING HUANG $^{1}$, SONG CAO $^{1}$, ZHUANG-JIANG LI ${ }^{1}$ and LONG-JUN DAI ${ }^{1,4}$ \\ ${ }^{1}$ Guangxi Key Laboratory for Transplantation Medicine, Institute of Transplant Medicine; ${ }^{2}$ Guangxi Transplantation Medicine \\ Research Center of Technology; ${ }^{3}$ Department of Organ Transplantation in Guangzhou Military Region, \\ 303 Hospital of People's Liberation Army, Nanning, Guangxi 530021, P.R. China; \\ ${ }^{4}$ Department of Surgery, University of British Columbia, Vancouver, BC V5Z 1L8, Canada
}

Received July 2, 2015; Accepted November 18, 2015

DOI: $10.3892 /$ etm.2016.3001

\begin{abstract}
Effective use of all available donated organs is critical, in order to meet the increasing demand for transplants. The present study explored liver transplantation with livers that were donated following cardiac death (DCD). According to the guidelines established by The Red Cross Society of China, 42 DCD organs were procured. Selected donors were treated with extracorporeal membrane oxygenation (ECMO) prior to the organ retrieval. The present single-center study included 6 liver transplantations of DCD organs (5 liver transplants and 1 liver-kidney combined transplant). All 6 recipients had a successful recovery without significant complications. The serum alanine transaminase, total bilirubin and international normalized ratio returned to the normal levels within a short period of time following transplantation, and the liver function remained normal during the follow-up period, which lasted up to 24 months. The present report demonstrated the feasibility of orthotopic liver transplantation using DCD livers. The pre-conditioning DCD donors and optimization of the recipient's condition using ECMO, played a crucial role in ensuring the success of transplantation.
\end{abstract}

\section{Introduction}

Chronic liver disease is considered a major cause of mortality worldwide, and particularly in Asia (1). The majority of

Correspondence to: Dr Xu-Yong Sun, Gaungxi Key Laboratory for Transplantation Medicine, Institute of Transplant Medicine, 303 Hospital of People's Liberation Army, 52 Plant Road, Nanning, Guangxi 530021, P.R. China

E-mail: xuyongsun303@gmail.com

Dr Long-Jun Dai, Department of Surgery, University of British Columbia, 400-828 West 10th Avenue, Vancouver, BC V5Z 1L8, Canada

E-mail: ljdai@mail.ubc.ca; longjundai@gmail.com

Key words: donation after cardiac death, liver transplantation, hepatic cirrhosis chronic liver diseases are initiated by hepatitis B virus (HBV) and hepatitis $\mathrm{C}$ virus (HCV) infection $(2,3)$. More than half a million people with $\mathrm{HBV}$ infection die annually from complications of chronic hepatitis B, such as hepatic cirrhosis and liver cancer (4). Liver transplantation has been shown to be the most effective therapy for patients with advanced liver disease (5). Transplantation offers patients, whose lives have been severely restricted or almost succumbed to the disease, a marked improvement in their health and well-being; however, the demand for organ transplants outreaches the number of donated organs (6). Organ donation after cardiac death (DCD) could maximize the use of these scarce resources. Previously known as non-heart-beating organ donation, DCD has been an end-of-life option for $>30$ years in the USA and Europe (7). DCD is performed in cases where life support is withdrawn from a patient after a physician has determined that there is no long-term prognosis for recovery, or in patients who do not meet the criteria for neurological death. The option of DCD should be offered prior to life support withdrawal, but only after the decision to withdraw has been reached (8).

Initially, DCD presented various limitations, such as poor organ function following transplantation. However, advances in medication and surgical techniques have markedly improved the outcomes for recipients of DCD organs. These advances, along with the severe shortage of donated organs promote DCD as an alternative for patients and families (8).

The use of DCD organs has rapidly developed in China since the establishment of DCD standard by the Red Cross Society and Ministry of Health of China in 2010 (9). The aim of the present study was to document successful liver transplantation using DCD organs and promote the development of these transplantation techniques in China.

The present single-centre study reports 6 liver transplantations performed with DCD organs, including 5 liver transplants and 1 liver-kidney combined transplant.

\section{Case report}

Between January 2011 and December 2013, 6 liver transplantations (including 1 liver-kidney and 5 liver transplants) were performed with DCD organs at the 303 Hospital of People's 
Table I. Recipient information.

\begin{tabular}{lccccc}
\hline Recipient & Age, years & Gender & APACHE II score & MELD & $\begin{array}{c}\text { Estimated } \\
\text { mortality, \% }\end{array}$ \\
\hline 1 & 42 & Male & 7 & 9 & 8 \\
2 & 35 & Male & 14 & 38 & 12 \\
3 & 37 & Male & 9 & 41 & 15 \\
4 & 40 & Male & 11 & 34 & 11 \\
5 & 47 & Male & 12 & 37 & 9 \\
6 & 43 & Male & 6 & 13 & 25 \\
\hline
\end{tabular}

APACHE II, acute physiology and chronic health evaluation II; MELD, model for end-stage liver disease.

Table II. Donor information.

\begin{tabular}{lcccccc}
\hline Donor & Age, years & Gender & Cause of mortality & UW score & WI length, min & CI, h \\
\hline 1 & 32 & Male & Traumatic brain injury & 21 & 5.6 & 6.8 \\
2 & 30 & Male & Cerebral hemorrhage & 22 & 6.1 & 8.3 \\
3 & 37 & Male & Traumatic brain injury & 23 & 6.7 & 9.5 \\
4 & 53 & Male & Cerebral hemorrhage & 22 & 7.9 & 7.8 \\
5 & 42 & Male & Traumatic brain injury & 23 & 8.4 & 8.1 \\
6 & 43 & Male & Traumatic brain injury & 21 & 9.5 & 9.5 \\
\hline
\end{tabular}

UW, University of Wisconsin score; WI, warm ischemia; CI, cold ischemia.

Liberation Army (Nanning, China), with the approval of the Medical Ethics Committee at this institution. Written informed consent was obtained from all patients. Transplant decisions were made according to the availability of donors, blood type and cross-matching. The 6 transplant recipients were males aged 35-47 years, who suffered from cirrhosis-induced hepatic decompensation (3 cases), primary liver cancer (2 cases) or hepatic decompensation combined with renal failure (1 case). Table I lists information obtained from the evaluation of the recipients.

Acute physiology and chronic health evaluation II (APACHE II) scoring system and the model for end-stage liver disease (MELD) were used to evaluate the severity of liver disease in the recipients. APACHE II point scores are based upon values of 12 routine physiologic measurements, age and previous health status, in order to provide a general measure of the severity of disease (10). MELD predicts liver disease severity based on serum creatinine, serum total bilirubin and international normalized ratio (INR) (11).

In total,42 DCD livers were collected between January 2011 and December 2013 at the 303 Hospital of People's Liberation Army from male patients aged $30-53$ years, who succumbed to cerebral hemorrhage (13 cases) or traumatic brain injury (29 cases). The donation procedure followed the DCD guidelines of China (9). Of these, 32 donor livers were not procured due to alcoholic cirrhosis, liver cancer, hepatic distomiasis and long durations of hepatic warm ischemia (>30 min). Therefore, 10 DCD livers were successfully procured for transplantation, 4 of which were distributed to other centers through the Red
Cross Society of China (Beijing, China). Transplantations of the remaining 6 livers were performed at the 303 Hospital of People's Liberation Army.

Preoperative evaluation was performed on all donors, and were found to be negative for human immunodeficiency virus, hepatitis B surface antigen, HCV antibody and syphilis antibody (12). The blood oxygenation status and hemodynamics were maintained by the intensive care unit (ICU) group. Based on the stability of hemodynamics and blood oxygenation status, 5 donors were further supported with extracorporeal membrane oxygenation (ECMO) for 6-10 h prior to organ retrieval. The 6 donors underwent controlled cardiac death, with planned withdrawal of ventilatory and organ-perfusion support in the face of catastrophic illness (Maastricht classification class III; 13). The University of Wisconsin score (UW; 14) was found to be 21-23 (Table II). The cardiac death occurred immediately following the withdrawal of life support, and the warm ischemia time was kept to within $10 \mathrm{~min}$. Following the confirmation of cardiac death, the DCD organs were procured by a combined abdominal organ retrieval procedure (15). Table II lists the relevant information of all 6 donors.

Orthotopic liver transplantation (OLT) was then performed in the 6 liver transplant recipients. Liver transplantation by classical caval reconstruction was performed on 3 of the recipients (nos. 1, 3 and 6; Table I), while 2 recipients (nos. 2 and 4) received non-bypass OLT and 1 recipient (no. 5) underwent the piggy-back technique.

The immunity of the recipient was suppressed through the induction of rabbit anti-human $\mathrm{T}$ lymphocyte immuno- 


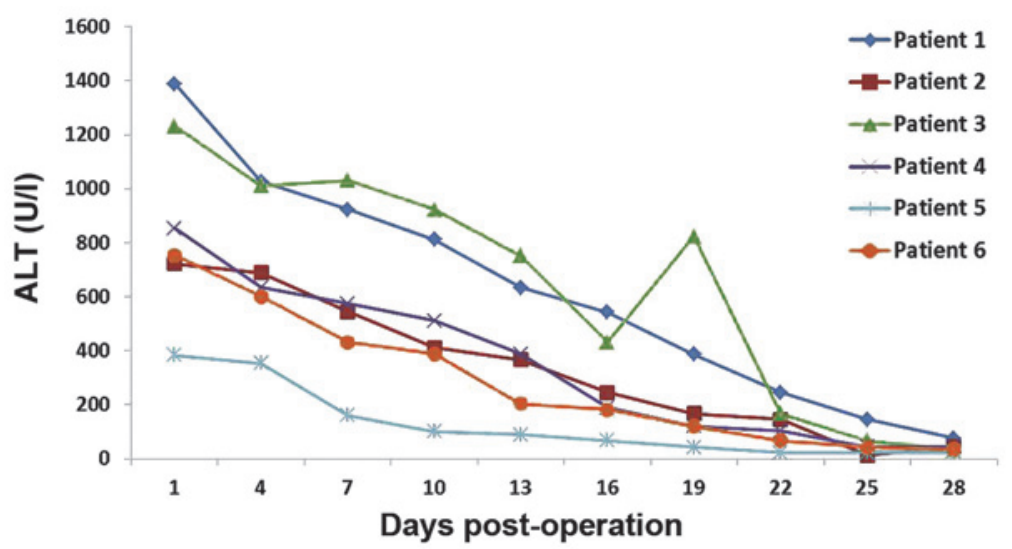

Figure 1. Dynamic observation of serum ALT. ALT, alanine transaminase.

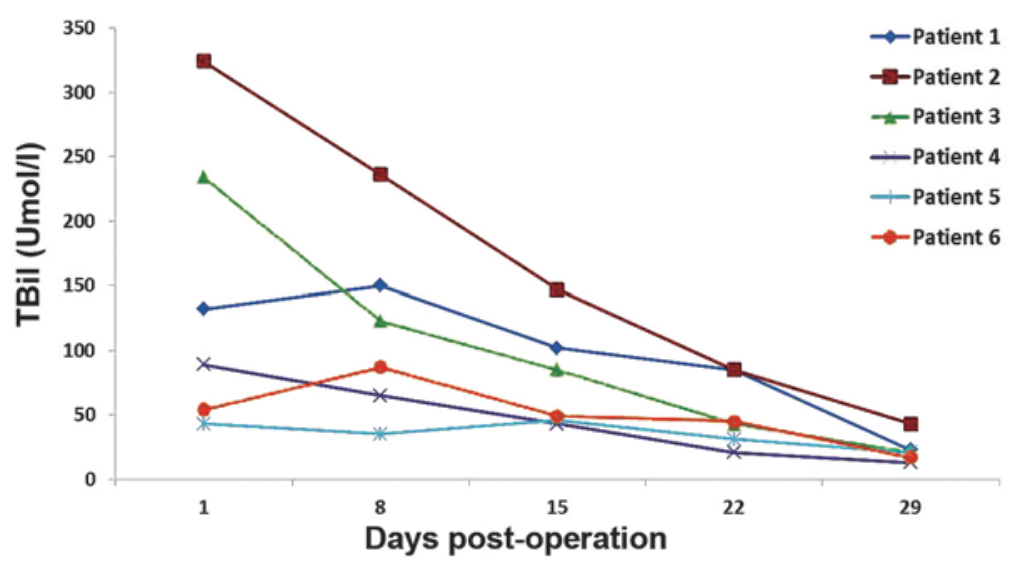

Figure 2. Changes in blood TBil. TBil, total bilirubin.

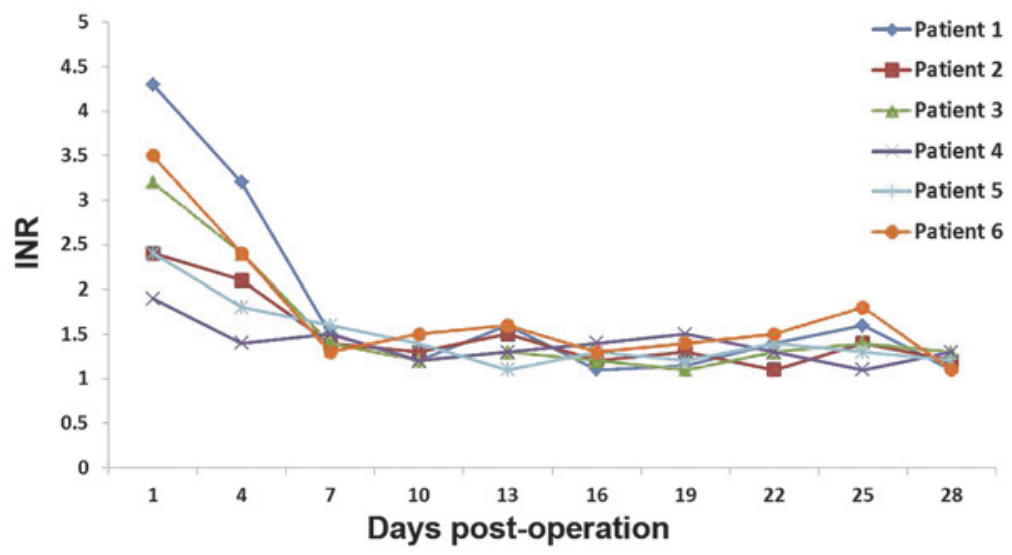

Figure 3. Measurements of INR. INR, international normalized ratio.

globulinG CD25 polyclonal antibody $(100 \mathrm{mg} / 5 \mathrm{ml}$ saline; S20090069; Fresenius Biotech GmBH, Grafelfing, Germany), followed by tacrolimus $(0.15 \mathrm{mg} / \mathrm{kg}$ per day; Astellas Pharma China,Inc., Shanghai, China) and mycophenolate mofetil (3 mg/ day; Roche Diagnostics, Shanghai, China). Postoperatively, all patients were maintained in the ICU for 5-10 days and received routine monitoring of vital signs and organ functions. The anastomotic sites of the transplanted livers were regularly monitored using color Doppler sonography. With regard to the patient that received the liver-kidney combined transplant, the renal artery resistance index and renal blood supply of the transplanted kidney were regularly examined. Recipients were administered $0.15 \mathrm{mg} / \mathrm{kg}$ micafungin sodium (Astellas Pharma China, Inc.) daily for the first 7-14 days following transplantation in order to prevent and treat inflammation.

The 6 recipients recovered considerably well without significant complications, such as primary graft failure, thrombosis, graft rejection or biliary stricture. The dynamic changes 
of serum alanine transaminase (ALT; Fig. 1), total bilirubin (TBil; Fig. 2) and INR (Fig. 3) were routinely recorded. The patient that received the liver-kidney combined transplant presented normal urine levels immediately following surgery $(2,500$ mlday $)$ and the serum creatinine $(\mathrm{Cr})$ levels had returned to the normal values within 7 days. All liver recipients were discharged from the hospital within 20-30 days after the transplantation. Their liver function remained normal during the entire follow-up period, which lasted up to 24 months.

\section{Discussion}

At present, the data for the long-term outcomes of OLT using DCD livers are controversial. Certain studies using pooled registry data $(16,17)$ have reported inferior transplant outcomes of DCD compared with donation following brain death (DBD), while single-center studies have shown comparable survival outcomes for the two types of organ donation $(18,19)$. Since a DBD program has not yet been established in China, the use of DCD as a strategy to increase the limited availability of donor livers is critical.

In the present study, controlled class III cardiac death was observed in the 6 donors. The relatively short warm ischemia time was ensured by a high-risk UW score following the withdrawal of life support. The warm ischemia time of these 6 donors was found to be $<10 \mathrm{~min}$. Recent reports have indicated that the survival of DCD grafts, as well as their recipients, are significantly affected by complications, including primary non-function, hepatic artery thrombosis and ischemic cholangiopathy $(20,21)$. Such complications, however, did not occur in any of the 6 recipients of the present study. Recipient 2 exhibited a high TBil, which may be associated with his preoperative status (MELD 38) or the quality of the transplanted liver. MELD is widely used to predict the severity of liver disease and is proportionally correlated with mortality risk (11). The high TBil of recipient 3 was due to the moderate steatosis observed in the DCD liver. A rapid decease in TBil was observed in recipients $1,4,5$ and 6 , who had low MELD scores and did not present liver steatosis. In recipients 2 and 3, the increase in INR may have been due to the high MELD scores and presence of liver steatosis.

The use of fatty livers (with steatosis) as grafts is relatively risky, since it has been found that the incidence of primary non-function in transplant recipients with liver steatosis is considerably higher compared with that in patients with normal livers (22). The early malfunction of grafted livers is proportionally associated with the extent of liver steatosis (23). A comparative study on the 3-month survival rate following liver implantation, length of stay in the ICU and hospital, as well as Child-Pugh evaluation (24) and liver function, demonstrated that the prognosis was not significantly affected by mild (10-30\%) or moderate (30-60\%) steatosis of the grafted liver (25). Kwon et al (26) analyzed 245 liver transplants with mild steatosis between 1999 and 2005, and divided the liver recipients into three groups, according to the degree of liver steatosis (the $0-9,10-19$ and $20-30 \%$ groups). No statistically significant differences were observed in detected parameters, including length of surgery, blood loss, peak value of TBil, serum aspartate aminotransferase and incidence of complications, among the three groups. The results were similar to those observed in 30 and 50\% alcoholic cirrhosis livers (27). In the present study, 3 liver transplant recipients with moderate hepatic steatosis (recipients 4,5 and 6) exhibited high ALT and TBil levels, which returned to the normal levels on day 17 following transplantation. In patients with normal liver grafts (recipients 1 and 2), ALT reached 200-400 Ul and returned to its normal levels on days 7 (recipient 1 ) and 9 (recipient 2) following transplantation. Notably, recipient 3 exhibited a fast recovery of liver function without any indication of hepatic steatosis.

China is of the counties with the highest demands of liver transplants worldwide, mainly due to the extremely high incidence of HBV and liver cancer (28). The appropriate application of DCD may be able to maximize the use of these scarce resources, in order to save more lives. In the present study, the transplantation of DCD livers with moderate steatosis indicated that controlled class III, high UW score and short warm ischemia time are essential factors in determining the success of transplantation. ECMO was able to maintain steady hemodynamics and high blood oxygenation, thereby improving donor liver function prior to organ retrieval, which played an important role in the successful short- and long-term outcomes of liver transplantation.

\section{Acknowledgements}

This study was supported by grants from the Guangxi Natural Science Foundation (no. 2013GXNSFAA019253) and Guangxi Science and Technology Development Project (no. 14124003-8).

\section{References}

1. Wang FS, Fan JG, Zhang Z, Gao B and Wang HY: The global burden of liver disease: The major impact of China. Hepatology 60: 2099-2108, 2014.

2. Lai CL, Ratziu V, Yuan MF and Poynard T: Viral hepatitis B. Lancet 362: 2089-2094, 2003.

3. Poynard T, Yuan MF, Ratziu V and Lai CL: Viral hepatitis C. Lancet 362: 2095-2100, 2003.

4. Cholongitas E, Tziomalos K and Pipili C: Management of patients with hepatitis B in special populations. World J Gastroenterol 21: 1738-1748, 2015.

5. Watt KD: Keys to long-term care of the liver transplant recipient. Nat Rev Gastroenterol Hepatol 12: 639-648, 2015.

6. Hackl C, Schlitt HJ, Kirchner GI, Knoppke B and Loss M: Liver transplantation for malignancy: Current treatment strategies and future perspectives. World J Gastroenterol 20: 5331-5344, 2014.

7. Morrissey PE and Monaco AP: Donation after circulatory death: current practices, ongoing challenges, and potential improvements. Transplantation 97:258-264, 2014.

8. Manara AR, Murphy PG and O'Callaghan G: Donation after circulatory death. Br J Anaesth 108 (Suppl 1): i108-i121, 2012.

9. Liu Y: Establishment of human organ donation system in China - resolving the issues of organ sources and standardizing the procedures of organ transplantation. Zhong Hua Qi Guan Yi Zhi Za Zhi 31: 390-392, 2010 (In Chinese).

10. Knaus WA, Draper EA, Wagner DP and Zimmerman JE: APACHE II: A severity of disease classification system. Crit Care Med 13: 818-829, 1985.

11. Wiesner R, Edwards E, Freeman R, Harper A, Kim R, Kamath P, Kremers W, Lake J, Howard T, Merion RM, et al; United Network for Organ Sharing Liver Disease Severity Score Committee: Model for end-stage liver disease (MELD) and allocation of donor livers. Gastroenterology 124: 91-96, 2003.

12. Liu YF: Assessment of organs from cardiac death donors and in vitro preparation. Zhong Hua Qi Guan Yi Zhi Za Zhi 31: 393-396, 2010 (In Chinese).

13. Kootstra G, Daemen JH and Oomen AP: Categories of non-heart-beating donors. Transplant Proc 27: 2893-2894, 1995. 
14. Lewis J, Peltier J, Nelson H, Snyder W, Schneider K, Steinberger D, Anderson M, Krichevsky A, Anderson J,Ellefson J and D'Alessandro A.: Development of the University of Wisconsin donation After Cardiac Death Evaluation Tool. Prog Transplant 13: 265-273, 2003.

15. Issa $S$ and Al-Bishri S: The role of early identification of superior mesenteric artery in a modified technique for retrieval of abdominal organs for transplantation. Saudi J Kidney Dis Transpl 19: 389-396, 2008.

16. Johnson RJ, Bradbury LL, Martin K and Neuberger J; UK Transplant Registry: Organ donation and transplantation in the UK-the last decade: A report from the UK national transplant registry. Transplantation 97 (Suppl 1): S1-S27, 2014.

17. Callaghan CJ, Charman SC, Muiesan P, Powell JJ, Gimson AE and van der Meulen JH; UK Liver Transplant Audit: Outcomes of transplantation of livers from donation after circulatory death donors in the UK: A cohort study. BMJ Open 3: e003287, 2013.

18. Dubbeld J, van Hoek B and Ringers J: Use of a liver from donor after cardiac death: Is it appropriate for the sick or the stable? Curr Opin Organ Transplant 16: 239-242, 2011

19. Grewal HP, Willingham DL, Nguyen J, Hewitt WR, Taner BC Cornell D, Rosser BG, Keaveny AP, Aranda-Michel J, Satyanarayana $\mathrm{R}$, et al: Liver transplantation using controlled donation after cardiac death donors: An analysis of a large single-center experience. Liver Transpl 15: 1028-1035, 2009.

20. Jay C, Ladner D, Wang E, Lyuksemburg V, Kang R, Chang Y, Feinglass J, Holl JL, Abecassis M and Skaro AI: A comprehensive risk assessment of mortality following donation after cardiac death liver transplant - an analysis of the national registry. J Hepatol 55: 808-813, 2011.
21. Foley DP, Fernandez LA, Leverson G, Anderson M, Mezrich J, Sollinger HW and D'Alessandro A: Biliary complications after liver transplantation from donation after cardiac death donors: An analysis of risk factors and long-term outcomes from a single center. Ann Surg 253: 817-825, 2011.

22. Neuberger J: Transplantation: Assessment of liver allograft steatosis. Nat Rev Gastroenterol Hepatol $10: 328-329,2013$.

23. Ureña MA, Ruiz-Delgado FC, González EM, Segurola CL, Romero CJ, García IG, González-Pinto I and Gómez Sanz R: Assessing risk of the use of livers with macro and microsteatosis in a liver transplant program. Transplant Proc 30: 3288-3291, 1998.

24. Pugh RN, Murray-Lyon IM, Dawson JL, Pietroni MC and Williams R: Transection of the oesophagus for bleeding oesophageal varices. Br J Surg 60: 646-649, 1973

25. Nikeghbalian S, Nejatollahi SM, Salahi H, Bahador A, Sabet B, Jalaeian H, Geramizadeh B, Dehghani SM and Malek-Hosseini SA: Does donor's fatty liver change impact on early mortality and outcome of liver transplantation? Transplant Proc 39: 1181-1183, 2007.

26. Kwon CH, Joh JW, Lee KW, Kim SJ, Han YS, Park JW, Kim DJ, Park JB and Lee SK: Safety of donors with fatty liver in liver transplantation. Transplant Proc 38: 2106-2107, 2006.

27. Cho JY, Suh KS, Kwon CH, Yi NJ, Kim MA, Jang JJ, Minn KW and Lee KU: Auxiliary partial orthotopic living donor liver transplantation in a patient with alcoholic liver cirrhosis to overcome donor steatosis. Transpl Int 19: 424-429, 2006.

28. Chen JG and Zhang SW: Liver cancer epidemic in China: Past, present and future. Semin Cancer Biol 21: 59-69, 2011. 